\title{
Penehyclidine hydrochloride exerts protective effects in rats with acute lung injury via the Fas/FasL signaling pathway
}

\author{
QIAN KONG ${ }^{1 *}$, XIAOJING WU ${ }^{1 *}$, WEINA DUAN ${ }^{1}$, LIYING ZHAN $^{1}$ and XUEMIN SONG ${ }^{2}$ \\ ${ }^{1}$ Department of Anesthesiology, Renmin Hospital of Wuhan University, Wuhan, Hubei 430060; \\ ${ }^{2}$ Department of Anesthesiology and Critical Care Medicine, Zhongnan Hospital of Wuhan University, \\ Wuhan, Hubei 430071, P.R. China
}

Received June 14, 2018; Accepted January 1, 2019

DOI: $10.3892 /$ etm.2019.7340

\begin{abstract}
Acute lung injury (ALI) is a critical syndrome that is associated with high morbidity and mortality rates. The activation of the Fas/Fas ligand (FasL) signaling pathway may be an important pathophysiological mechanism during ALI development. Penehyclidine hydrochloride (PHC) has been revealed to exhibit anti-apoptotic properties and may attenuate the observed systemic inflammatory response. The present study was performed to elucidate the molecular mechanism of $\mathrm{PHC}$ in the regulation of the Fas/FasL signaling pathway in rats with ALI. An ALI rat model was constructed by inducing blunt chest trauma and hemorrhagic shock (T/HS), with PHC administration prior to or following T/HS. At $6 \mathrm{~h}$ following T/HS, blood samples and lung tissues were collected. Western blotting, arterial blood gas analysis, ELISA, hematoxylin and eosin staining, terminal deoxynucleotidyl transferase-mediated dUTP nick-end labeling staining and biochemical indicator analysis were performed to determine the degree of lung injury and the key signaling pathways associated with lung damage. The results indicated that the administration of PHC following T/HS effectively attenuates lung injury by improving pulmonary oxygenation, decreasing histopathological damage, decreasing polymorphonuclear neutrophil count and decreasing Fas, FasL, caspase-8, caspase-3, tumor necrosis factor- $\alpha$, interleukin (IL)- 6 and IL-1 $\beta$ expression.
\end{abstract}

Correspondence to: Professor Xuemin Song, Department of Anesthesiology and Critical Care Medicine, Zhongnan Hospital of Wuhan University, 169 Donghu Road, Wuchang, Wuhan, Hubei 430071, P.R. China

E-mail: sxmc11018@163.com

*Contributed equally

Abbreviations: ALI, acute lung injury; PHC, penehyclidine hydrochloride; ARDS, acute respiratory distress syndrome; T/HS, blunt chest trauma and hemorrhagic shock

Key words: penehyclidine hydrochloride, Fas, Fas ligand, apoptosis, acute lung injury
The results indicated that PHC exhibits anti-apoptotic functions and exerts protective effects in ALI rats induced by $\mathrm{T} / \mathrm{HS}$, which may be attributed to the inhibition of the Fas/FasL signaling pathway.

\section{Introduction}

Blunt chest trauma and hemorrhagic shock (T/HS) occurs in patients with poly-trauma (possibly following vehicular accidents) and is a leading risk factor in the development of acute lung injury (ALI) and acute respiratory distress syndrome (ARDS) $(1,2)$. T/HS exhibits high morbidity and mortality rates $(3,4)$. Furthermore, it is well known that apoptosis is involved in distinct types of ALI. Experimentally, Messer et al (5) and Thakkar et al (6) demonstrated that the activation of apoptosis via the Fas/Fas ligand (FasL) signaling pathway was important for the development of ALI caused by trauma or hemorrhagic shock. Clinically, Glavan et al (7) revealed that the content of soluble Fas and FasL was increased in pulmonary edema fluid, which was strongly associated with increased morbidity and mortality in patients with ALI. Additionally, Herrero et al (8) determined that the inhibition of the Fas/FasL signaling pathway alleviated ALI/ARDS damage.

Penehyclidine hydrochloride (PHC) is a novel anti-cholinergic drug that was first developed by the Beijing Institute of Pharmacology and Toxicology, Academy of Military Medical Sciences (Beijing, China), which can be applied in anti-apoptosis and anti-inflammation treatment $(9,10)$. Recently, an increasing number of studies have indicated that PHC may alleviate lung injuries by inhibiting ALI-induced apoptosis and inflammation (11-13). Furthermore, Wang et al (14) revealed that PHC mitigates lung injury by regulating the expression of Bcl-2-associated $\mathrm{X}$ (bax) and B-cell lymphoma-2 (Bcl-2) in a rat model of ALI following blunt chest trauma. Cui et al (15) also demonstrated that PHC pre-treatment reduced the expression of bax and caspase-3, decreased the indices of apoptosis and pulmonary vascular resistance, and improved $\mathrm{PaO}_{2} / \mathrm{FiO}_{2}$ and $\mathrm{Bcl}-2 /$ bax ratios in pigs with dichlorvos-induced ALI. However, the underlying mechanism of PHC in ALI requires further elucidation. The current study aimed to determine the effect of PHC on ALI as well as associated signaling pathways. 


\section{Materials and methods}

Animals. A total of 40 Male Sprague-Dawley rats (age, 8-10 weeks) with a body weight of 245-275 g were obtained from the Hunan Institute for Biologic Sciences (Hunan, China; certificate no. SCXX 2009-0004) under specific pathogen-free conditions. The current study was approved by Medical Ethics Committee of Renmin Hospital of Wuhan University (Wuhan, China) and was performed in accordance with the National Institutes of Health Guidelines for the Care and Use of Laboratory Animals. Animals were housed under a $12 \mathrm{~h} \mathrm{light/dark} \mathrm{cycle} \mathrm{at} \mathrm{a} \mathrm{temperature} \mathrm{of} 22^{\circ} \mathrm{C}$ and a humidity of $50-60 \%$ with free access to food and water.

T/HS model. Rats were anesthetized with intraperitoneal (IP) injections of sodium pentobarbital $(30 \mathrm{mg} / \mathrm{kg})$. To establish a rat model of blunt chest trauma, the current study generated an isolated bilateral lung contusion as described previously (16). Following anesthesia induction, a hollow cylinder (weight, $300 \mathrm{~g}$ ) was dropped from a defined height $(83.3 \mathrm{~cm})$. The cylinder was encased in a vertical stainless steel tube, which was positioned on a platform. The precordial shield directed the impact force bilaterally to the lungs to prevent cardiac trauma (impact energy, $2.45 \mathrm{~J}$ ). This experiment was performed by our laboratory according to a previous study by Raghavendran et al (17). A rat model of nonlethal hemorrhagic shock was then established based on a previous study (12). The femoral artery and vein were cannulated with polyethylene (PE-50) tubing, and blood flowed via tubing, which was attached to the monitor (IntelliVue MP40; Phillips Medical Systems B.V., Eindhoven, The Netherlands), until the average arterial blood pressure reached $35 \pm 5 \mathrm{~mm} \mathrm{Hg}$. This pressure was then maintained for $60 \mathrm{~min}$. Following a hypotensive period of $60 \mathrm{~min}$, rats were resuscitated via the transfusion of removed blood with Ringer's lactate solution [Baxter Healthcare (Tanjin) Company, Ltd., Tianjin, China; Composition, $6.0 \mathrm{~g} / 1 \mathrm{Na}^{+}, 6.0 ; 0.3 \mathrm{~g} / 1 \mathrm{~K}^{+} ; 0.2 \mathrm{~g} / \mathrm{l} \mathrm{Ca}^{2+}, 2 \mathrm{H}_{2} \mathrm{O}$ and $\mathrm{Cl}$ twice over a period of $60 \mathrm{~min}$. ALI was defined as a $\mathrm{PaO}_{2} / \mathrm{FiO}_{2}$ of $<300 \mathrm{~mm} \mathrm{Hg}$.

Experimental protocols. A total of 40 rats were randomly divided into 4 equal groups (each, $\mathrm{n}=10$ ): A sham group, a T/HS group, a $\mathrm{PHC} 1$ group and a PHC2 group. According to previous studies $(12,16)$, rats of the PHC1 group were infused with $2 \mathrm{mg} / \mathrm{kg}$ PHC $30 \mathrm{~min}$ prior to blunt chest trauma. PHC2 group rats were infused with $2 \mathrm{mg} / \mathrm{kg}$ PHC 60 min following hemorrhagic shock. The Sham and T/HS groups received the same volume $(0.5 \mathrm{ml})$ of $0.9 \%$ normal saline solution. Prior to blunt chest trauma, rats were anesthetized and the femoral artery and vein were cannulated with polyethylene (PE-50) tubing for continuous invasive pressure monitoring and to establish venous access. Rats in the sham group were subjected to the same experimental procedures, including the cannulation of femoral artery and vein, but without T/HS. All animals were sacrificed under deep anesthesia (50 mg/kg IP pentobarbital) and exsanguinated from the right carotid artery $6 \mathrm{~h}$ following T/HS. Blood and lung samples were then collected.

Arterial blood gas analysis. Following the induction of T/HS for $6 \mathrm{~h}$, rats were anesthetized with an IP injection of pentobarbital $(50 \mathrm{mg} / \mathrm{kg}$ ) and arterial blood was obtained from the right carotid artery $(1 \mathrm{ml}$ each). Samples were immediately analyzed using a blood gas analyzer (Abbott Point of Care Inc., Princeton, NJ, USA) for the determination of $\mathrm{PaO}_{2}$ and $\mathrm{PaO}_{2} / \mathrm{FiO}_{2}$.

Polymorphonuclear neutrophils (PMNs) and protein in $B A L F$. Following rat sacrifice, the trachea was cannulated and lavaged. Bronchoalveolar lavage fluid (BALF) was prepared by washing the lungs three times with $2 \mathrm{ml}$ PBS. BALF was centrifuged at $400 \mathrm{x} \mathrm{g}$ for $10 \mathrm{~min}$ at $4^{\circ} \mathrm{C}$ to pellet cells. BALF supernatant was then used for protein analysis. The cell pellet was resuspended in PBS and then the BALF cell counts were performed following trypan blue exclusion. An aliquot of pooled BALF $(50 \mu \mathrm{l})$ from each rat was diluted 1:1 with trypan blue dye (Thermo Fisher Scientific, Inc., Waltham, MA, USA) and the total number of cells was counted using a standard hemocytometer. To analyze differential cell counts, $100 \mu \mathrm{l}$ of BALF from each rat was centrifuged 1,000 x g for $10 \mathrm{~min}$ at $4^{\circ} \mathrm{C}$ using a Cytospin (Thermo Fisher Scientific, Inc.). After slides were dried, cells were fixed with $3 \%$ glutaraldehyde in PBS for $15 \mathrm{~min}$ at room temperature, and stained using Wright Stain solution (cat. no. 32857; Sigma-Aldrich, USA; incubated for $30 \mathrm{sec}$ at room temperature) according to the manufacturer's instructions, and then differential cell counts were obtained by manually counting 200 cells per rat as previously described (18). BALF protein content was determined via a bicinchoninic acid (BCA) assay and absorbance was measured at $595 \mathrm{~nm}$.

Hematoxylin and eosin $(H \& E)$ staining and acute lung injury score. Following animal sacrifice through bleeding from the right carotid artery at $6 \mathrm{~h}$ following T/HS, lung tissue samples were harvested immediately. Right middle-lung specimens were fixed using $10 \%$ formalin at room temperature for $24 \mathrm{~h}$, sectioned $(5 \mu \mathrm{m})$ and stained with H\&E (hematoxylin staining for $10 \mathrm{~min}$ and eosin staining for $2 \mathrm{~min}$; each at room temperature). Sections were observed under a light microscope with a magnification of $x 100$. Sections were then evaluated and graded for the presence of interstitial neutrophillic infiltrate, intra-alveolar hemorrhage and pulmonary edema with a light microscope (BX51; Olympus Corporation, Tokyo, Japan).

Western blotting. Western blot analysis was performed to determine Fas, FasL, caspase-3 and caspase-8 protein levels in rat lung tissues. Lung tissues were homogenized and lysed in lysis buffer [25 mM Tris- $\mathrm{HCl}$ ( $\mathrm{pH} 7.6$ ), $1 \% \mathrm{NP}-40,0.5 \%$ sodium deoxycholate and $0.1 \%$ sodium dodecyl sulfate). Protein concentration was determined using a BCA protein assay kit (Invitrogen; Thermo Fisher Scientific, Inc.) and equal quantities $(50 \mu \mathrm{g})$ of protein were loaded per lane on a $14 \%$ SDS-PAGE gel. Samples were separated electrophoretically and then transferred to polyvinylidene difluoride membranes. Subsequently, membranes were blocked with 5\% skimmed milk (Sigma-Aldrich; Merck KGaA, Darmstadt, Germany) in Tris-buffered saline containing 0.1\% Tween-20 (TBS-T) at room temperature for $2 \mathrm{~h}$ on a rotary shaker $(60 \mathrm{rpm})$. Samples were then incubated with the following primary antibodies overnight at $4^{\circ} \mathrm{C}$ : Rabbit anti-Fas $(1: 1,000$; cat. no. sc-21730; Santa Cruz Biotechnology, Inc., Dallas, TX, USA), anti-FasL 

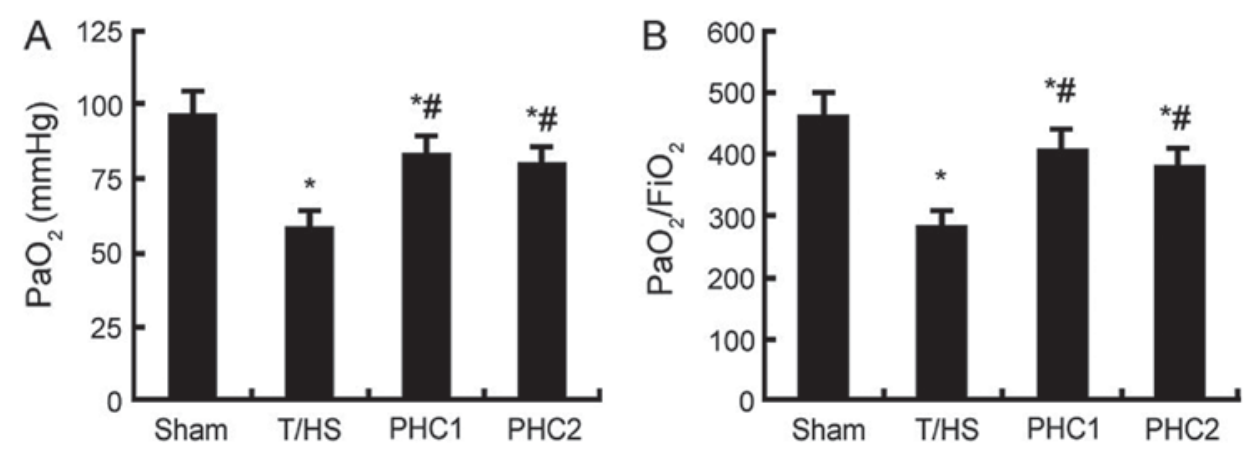

Figure 1. Effects of $\mathrm{PHC}$ on $\mathrm{PaO}_{2}$ and $\mathrm{PaO}_{2} / \mathrm{FiO}_{2}$. (A) $\mathrm{PaO}_{2}$ and (B) the $\mathrm{PaO}_{2} / \mathrm{FiO}_{2}$ ratio in the T/HS, $\mathrm{PHCl}$ and $\mathrm{PHC} 2$ group were significantly decreased compared with the sham group. $\mathrm{PaO}_{2}$ and $\mathrm{PaO}_{2} / \mathrm{FiO}_{2}$ ratios were also increased in the $\mathrm{PHC} 1$ and $\mathrm{PHC} 2$ groups compared with the T/HS group. Data are expressed as the mean \pm standard error of the mean. ${ }^{*} \mathrm{P}<0.05$ vs. the sham group; ${ }^{*} \mathrm{P}<0.05$ vs. the T/HS group. PHC, penehyclidine hydrochloride; T/HS, blunt chest trauma and hemorrhagic shock.

(1:1,000; cat. no. sc-834; Santa Cruz Biotechnology, Inc.), anti-caspase-3 (1:500; cat. no. 9662), anti-caspase-8 (1:500; cat. no. 9662; both Cell Signaling Technology, Inc., Danvers, MA, USA) and anti- $\beta$-actin (1:1,000; cat. no. ab8227; Abcam, Cambridge, UK). Subsequently, membranes were washed with TBS-T three times and incubated with peroxidase-conjugated secondary antibodies (1:2,000; cat. no. sc-2004; Santa Cruz Biotechnology, Inc.) for $1 \mathrm{~h}$ at room temperature. Membranes were then washed three times with TBST solution and the blotted protein bands were observed using enhanced chemiluminescence (Amersham; GE Healthcare Life Sciences, Little Chalfont, UK) and exposed to Kodak X-ray film (Kodak Biomax; cat. no. Z350400, Sigma-Aldrich; Merck KGaA). Proteins bands were quantified using Quantity One software (Bio-Rad, Hercules, CA, USA).

Immunohistochemistry. The expression of Fas and FasL were determined via immunohistochemistry. Lung tissues were fixed in $4 \%$ paraformaldehyde for $24 \mathrm{~h}$ at $4^{\circ} \mathrm{C}$, dehydrated, embedded in paraffin and subsequently cut into $5 \mathrm{~mm}$ slices. Sections were incubated with rabbit polyclonal anti-Fas (1:100; cat. no. sc-21730) and FasL (1:100; cat. no. sc-834; both Santa Cruz Biotechnology, Inc.) antibodies at $4^{\circ} \mathrm{C}$ for $15 \mathrm{~h}$. Samples were then incubated with horseradish peroxidase conjugated goat anti-rabbit immunoglobulin G (1:250; cat. no. sc-2004; Santa Cruz Biotechnology, Inc.) for $30 \mathrm{~min}$ at room temperature and diluted in blocking solution $(5 \%$ bovine serum albumin; cat. no. B2064; Sigma-Aldrich; Merck $\mathrm{KGaA}$ ) for $30 \mathrm{~min}$ at room temperature. Biotin-peroxide and diaminobenzidine were used as substrates for the color reaction. The mean optical density of Fas and FasL positive cells from each section were analyzed using image cytometry with HIPAS-2000 image analysis software (Wuhan Qianli Technical Imaging Co. Ltd., Wuhan, China). The number of positive microvessels in each section was counted in 10 microscopic fields (at magnification, $\mathrm{x} 400$ ) under a light microscope (BX51; Olympus Corporation, Tokyo, Japan). The specificity of immunohistochemical staining was tested using PBS at the same dilution. Tissue sections in the sham group were used as negative controls.

Terminal deoxynucleotidyl transferase-mediated dUTP nick-end labeling (TUNEL) assay. Apoptotic cells were stained via the TUNEL technique using an apoptosis detection kit (cat. no. APOAF-50TST; Sigma-Aldrich; Merck KGaA) following the manufacturer's protocol. The sections were added onto a coverslip with DPX mounting medium (cat. no. 06522; Sigma-Aldrich; Merck KGaA). The total number of cells and the number of positive cells were counted in two sections from each animal (at magnification, $\mathrm{x} 400$ ) in at least 10 fields of view in each section. The apoptosis index (AI) was calculated using the following formula: AI $(\%)=$ number of apoptotic cells/number of total cells $\times 100 \%$.

Cytokine measurement. The concentration of tumor necrosis factor- $\alpha$ (TNF- $\alpha$; cat. no. RK00027), interleukin (IL)-6 (cat. no. RK00008) and IL-1 $\beta$ (cat. no. RK00006) in lung tissue homogenate were measured using commercially available ELISA kits obtained from Abclonal Biotech Co., Ltd., Woburn, MA, USA) according to the manufacturer's protocol. The absorbance of each well was detected at $450 \mathrm{~nm}$ with a microplate reader. Each average value represents the values of triplicate experiments.

Statistical analysis. Data are presented as the mean \pm standard error of the mean and SPSS 17.0 software (SPSS, Inc., Chicago, IL, USA) was utilized to perform statistical analysis. Statistical comparisons between multiple groups were performed using one-way analysis of variance followed by a bonferroni post-hoc. $\mathrm{P}<0.05$ was considered to indicate a statistically significant difference.

\section{Results}

Effect of PHC on the $\mathrm{PaO}_{2}$ and $\mathrm{PaO}_{2} / \mathrm{FiO}_{2}$ of T/HS rats. As an evaluation index of gas exchange, $\mathrm{PaO}_{2} / \mathrm{FiO}_{2}$ was measured to estimate the degree of lung injury. Significant decreases in $\mathrm{PaO}_{2}$ (Fig. 1A) and the $\mathrm{PaO}_{2} / \mathrm{FiO}_{2}$ ratio (Fig. 1B) were observed in T/HS rats compared with the sham group. However, when compared with the T/HS group, pre-treatment or treatment with $\mathrm{PHC}$ efficiently increased the $\mathrm{PaO}_{2}$ and $\mathrm{PaO}_{2} / \mathrm{FiO}_{2}$ ratios in ALI rats $(\mathrm{P}<0.05)$.

Effect of PHC on PMNs and BALF protein concentration in $T / H S$ rats. PMNs are the primary inflammatory cells present in many diseases of the lung, including ALI/ARDS (19). 

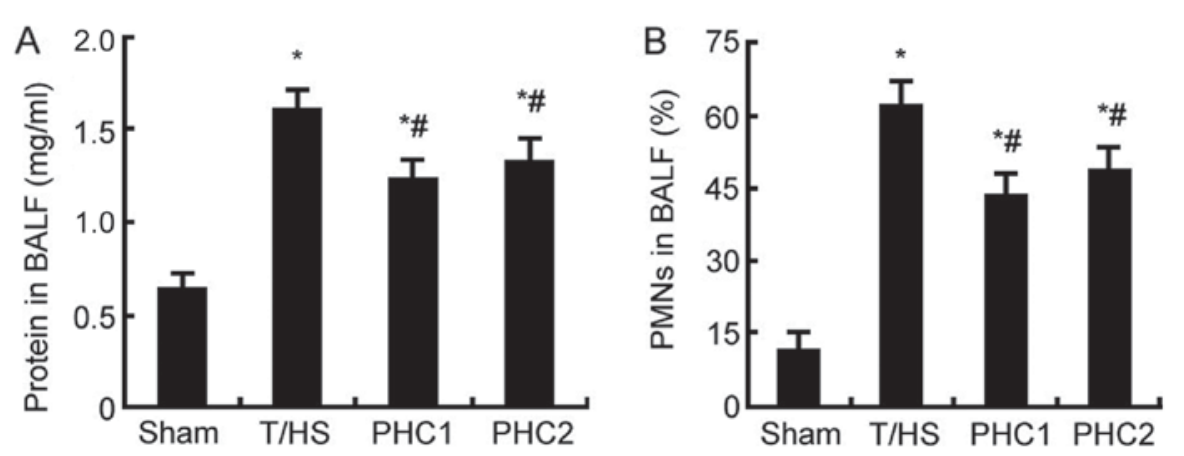

Figure 2. Effect of PHC on PMNs and protein concentration in BALF. The (A) protein concentration of and (B) the number of PMNs in BALF were assessed in the T/HS, PHC1 and PHC2 groups. The number of PMNs and the concentration of protein were decreased in the PHC1 and PHC2 groups compared with the T/HS group. Data are expressed as the mean \pm standard error of the mean. ${ }^{*} \mathrm{P}<0.05$ vs. the Sham group; ${ }^{*} \mathrm{P}<0.05$ vs. the T/HS group. PHC, penehyclidine hydrochloride; PMN, polymorphonuclear neutrophils; BALF, bronchoalveolar lavage fluid; T/HS, blunt chest trauma and hemorrhagic shock.

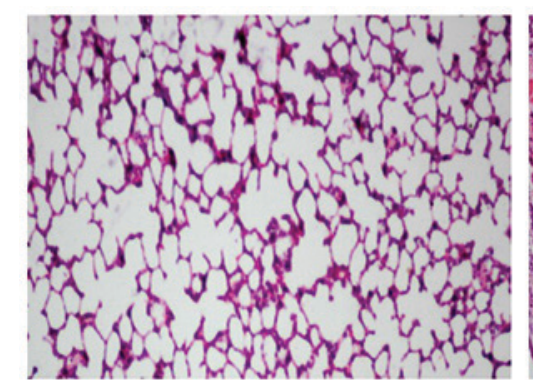

Sham

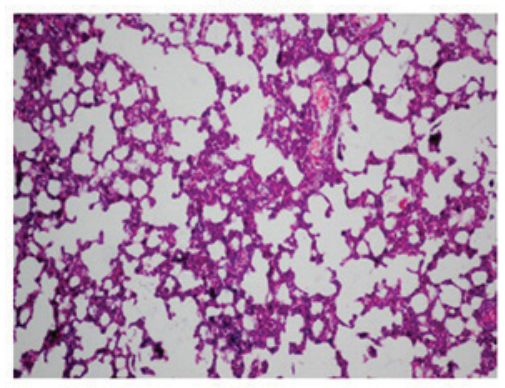

$\mathrm{PHC} 1$

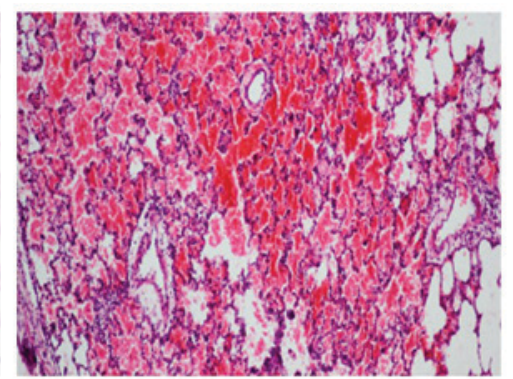

T/HS

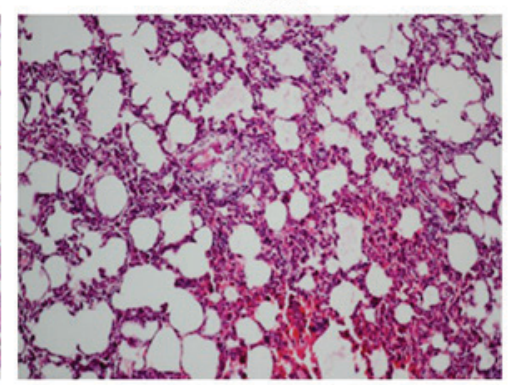

$\mathrm{PHC2}$

Figure 3. Effect of PHC on the lung histopathological changes observed in ALI rats. Hematoxylin and eosin staining revealed that severe hemorrhage and congestion, thickening of the alveolar wall, alveolar collapse and infiltration of alveoli with inflammatory cells was observed in the lung tissues of T/HS, PHC1 and PHC2 treated rats, compared with the sham group. However, compared with the T/HS group, lung injury was significantly ameliorated in the PHC1 and PHC2 groups. Original magnification, x100. PHC, penehyclidine hydrochloride; ALI, acute lung injury; T/HS, blunt chest trauma and hemorrhagic shock.

Furthermore, the concentration of protein in BALF is an indicator commonly used to detect pulmonary vascular permeability, which is an important characteristic of ALI/ARDS (20). When compared with the sham group, the concentration of protein (Fig. 2A) and the number of PMNs (Fig. 2B) in BALF were significantly increased $6 \mathrm{~h}$ following T/HS. However, PHC treatment prior to or following T/HS significantly reduced PMN infiltration and protein concentration compared with the T/HS group.

Effect of PHC on the histopathological changes observed in T/HS rats. To assess the effect of PHC on the histopathological changes observed in ALI rats, lung tissues from each group were subjected to $\mathrm{H} \& \mathrm{E}$ staining. As presented in Fig. 3, the sham group exhibited normal pulmonary histology with intact structures and clear pulmonary alveoli. By contrast, the tissue of the T/HS group exhibited serious damage, including severe hemorrhage and congestion, alveolar wall thickening, alveolar collapse and inflammatory cell infiltration into alveoli. However, the administration of PHC ameliorated pulmonary histopathological changes in ALI rats.

Effect of PHC on the expression of Fas, FasL, caspase-3 and caspase-8. Western blotting was performed to detect the level of Fas, FasL, caspase- 3 and caspase- 8 proteins. The results revealed that, in response to T/HS, the protein expression of Fas, FasL, caspase- 3 and caspase- 8 were significantly increased; while PHC treatment administered prior to or following T/HS induction significantly inhibited this increase (Fig. 4).

Effect of PHC on Fas and FasL protein expression in ALI lung tissue. Immunohistochemistry assays were performed to detect the protein expression of Fas and FasL. The results revealed that, when compared with the sham group, T/HS 

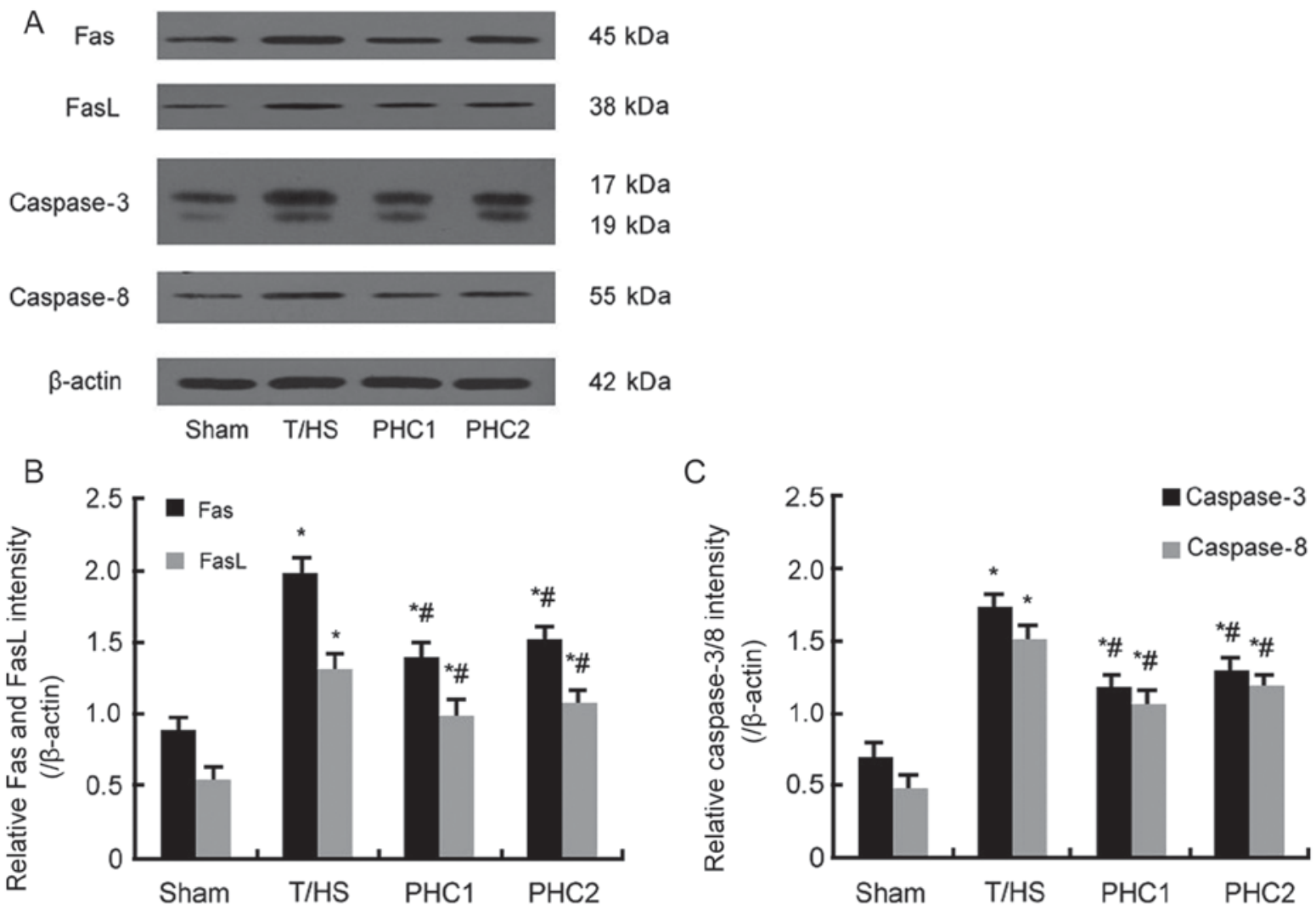

Figure 4. Effect of PHC on the expression of Fas, FasL, caspase-3 and caspase-8. (A) Western blotting was performed to assess protein levels of Fas, FasL, caspase-3 and caspase-8. (B) Statistical analysis of (B) Fas and FasL, and (C) caspase-3 and caspase-8. Increased levels of all proteins were observed in the lung tissue of T/HS, PHC1 and PHC2 treated rats when compared with the Sham group. However, protein levels were decreased in the PHC1 and PHC2 groups compared with the T/HS group. Data are expressed as mean \pm standard error of the mean. ${ }^{*} \mathrm{P}<0.05$ vs. the Sham group; ${ }^{\#} \mathrm{P}<0.05$ vs. the T/HS group. PHC, penehyclidine hydrochloride; FasL, Fas ligand; T/HS, blunt chest trauma and hemorrhagic shock.

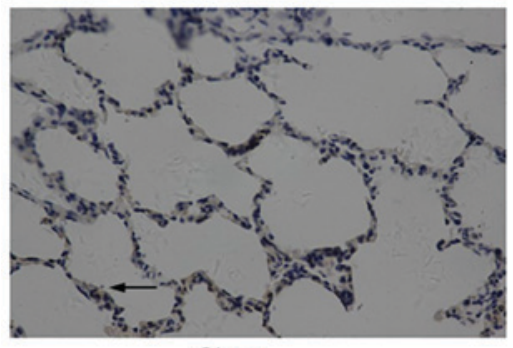

Sham

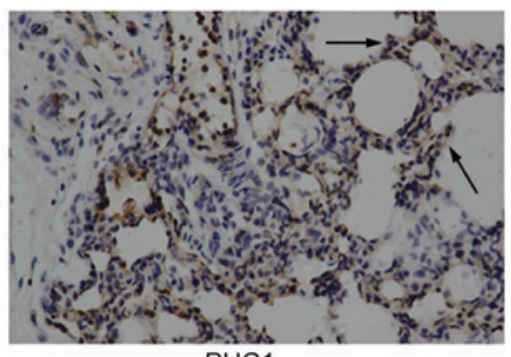

$\mathrm{PHC} 1$

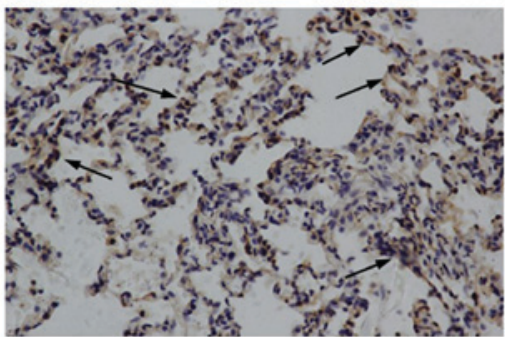

T/HS

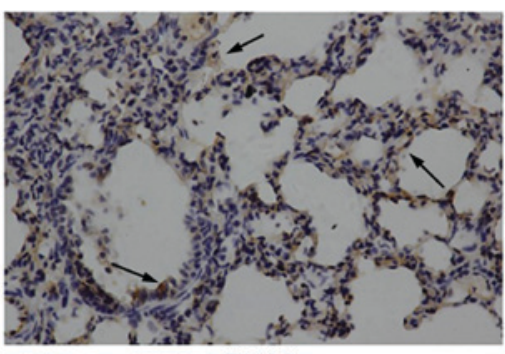

PHC2

Figure 5. Effect of PHC on Fas protein expression in the lung alveolar area of rats. In the T/HS, PHC1 and PHC2 groups, the protein levels of Fas were markedly increased compared with the sham group. However, Fas protein expression was decreased in the PHC1 and PHC2 groups compared with the T/HS group. Black arrows indicate Fas protein expression. PHC, penehyclidine hydrochloride; T/HS, blunt chest trauma and hemorrhagic shock.

induction markedly increased the protein levels of Fas (Fig. 5) and FasL (Fig. 6) in the alveolar area of the lung. Furthermore, the administration of PHC markedly reduced Fas and FasL levels compared with the T/HS group.
Effect of PHC on the degree of apoptosis in ALI rat lung tissue. As presented in Fig. 7, a small number of apoptotic cells were observed in the sham group (Fig. 7A), but apoptotic cells were significantly increased following T/HS 


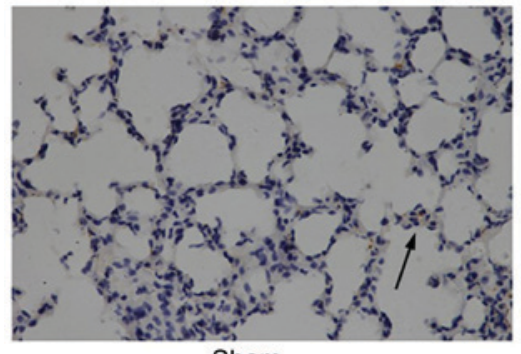

Sham

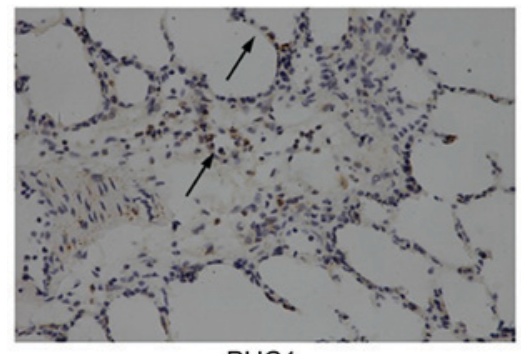

PHC1
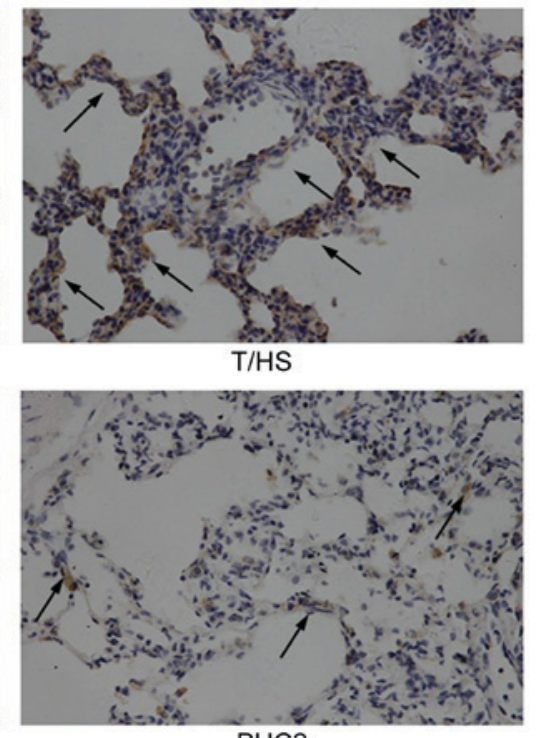

$\mathrm{PHC} 2$

Figure 6. Effect of PHC on FasL protein expression in lung tissue. In the T/HS, PHC1 and PHC2 groups, the protein levels of FasL in the alveolar area of the lung were markedly increased compared with that the sham group. Fas protein levels were however decreased in the PHC1 and PHC2 groups when compared with the T/HS group. Black arrows indicate FasL protein expression. PHC, penehyclidine hydrochloride; FasL, Fas ligand; T/HS, blunt chest trauma and hemorrhagic shock.

A

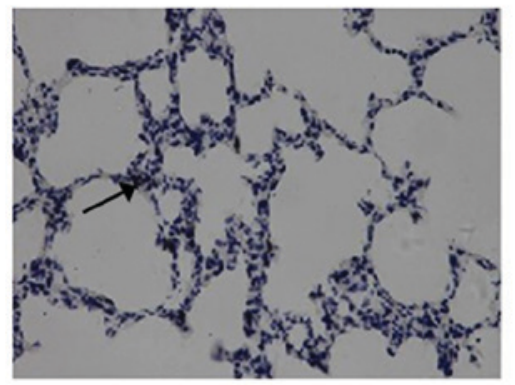

C

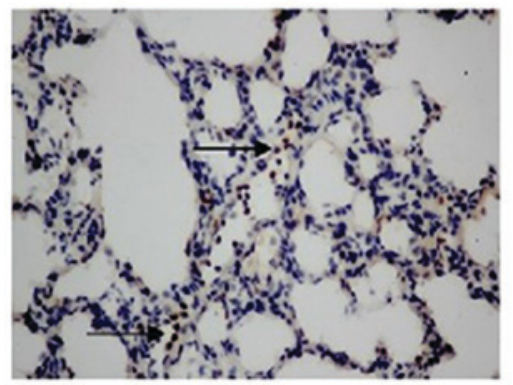

$B$

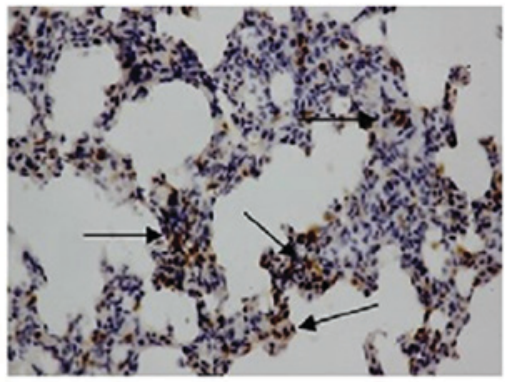

D

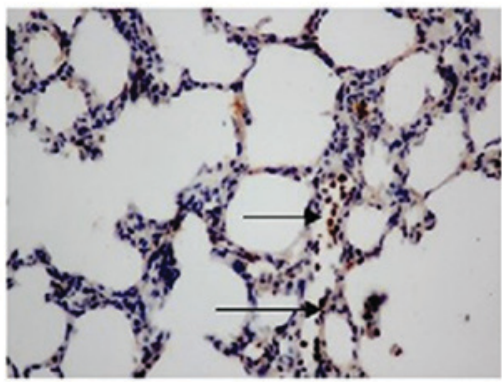

E

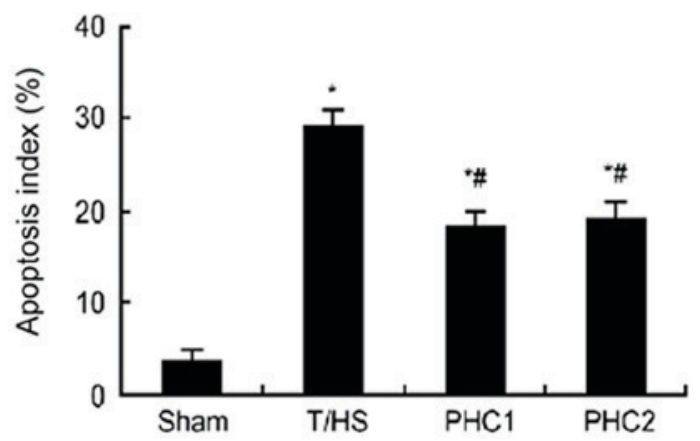

Figure 7. Effect of PHC on the degree of apoptosis in ALI rat lung tissue. A small number of apoptotic cells were observed in the (A) sham group. Compared with the sham group, apoptotic cells were significantly increased in the (B) T/HS, (C) PHC1 and (D) PHC2 groups. However, the number of apoptotic cells in the $\mathrm{PHCl}$ and $\mathrm{PHC} 2$ groups was decreased compared with the T/HS group. The (E) apoptosis index of lung tissues in T/HS rats was significantly increased compared with the sham group and the apoptosis index was statistically decreased in the PHC1 and PHC2 groups compared with the T/HS group. Black arrows indicate apoptotic cells. Data are expressed as mean \pm standard error of the mean. ${ }^{*} \mathrm{P}<0.05$ vs. the Sham group; ${ }^{*} \mathrm{P}<0.05$ vs. the T/HS group. PHC, penehyclidine hydrochloride; ALI, acute lung injury; T/HS, blunt chest trauma and hemorrhagic shock. 


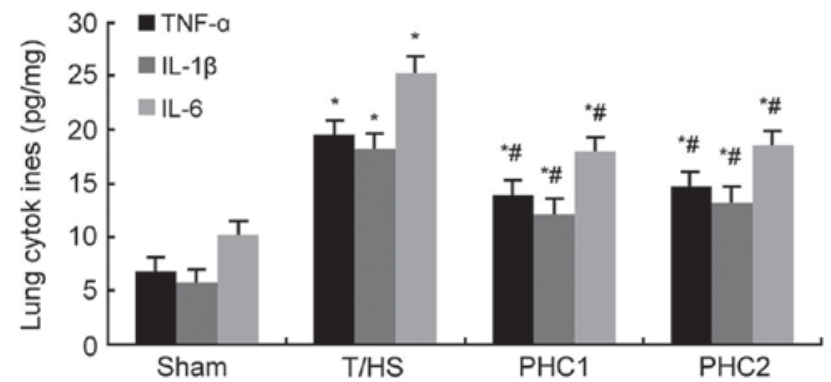

Figure 8. Effect of PHC on TNF- $\alpha$, IL-6 and IL-1 $\beta$ levels in ALI rat lung tissue. Compared with the sham group, the levels of TNF- $\alpha$, IL-1 $\beta$ and IL-6 in lung tissues were significantly increased in the T/HS, PHC1 and PHC2 groups. Compared with the T/HS group, the levels of thee cytokines were significantly decreased in the $\mathrm{PHC} 1$ and $\mathrm{PHC} 2$ groups. Data are expressed as mean \pm standard error of the mean. ${ }^{*} \mathrm{P}<0.05$ vs. the Sham group; ${ }^{\#} \mathrm{P}<0.05$ vs. the T/HS group. PHC, penehyclidine hydrochloride; TNF- $\alpha$, tumor necrosis factor- $\alpha$; IL, interleukin; ALI, acute lung injury; T/HS, blunt chest trauma and hemorrhagic shock.

induction (Fig. 7B, C and D). Compared with the T/HS group, the number of apoptotic cells in the $\mathrm{PHC} 1$ and $\mathrm{PHC} 2$ groups was decreased (Fig. 7C and D). Concomitantly, the apoptosis index of T/HS rat lungs was significantly increased compared with the sham group and the apoptosis index was statistically decreased following the administration of PHC (Fig. 7E).

Effect of PHC on TNF- $\alpha, I L-6$ and IL-1 $\beta$ levels. Lung tissues were collected $6 \mathrm{~h}$ following T/HS induction and the levels of cytokines within were measured using ELISA. The results demonstrated that the levels of TNF- $\alpha$, IL- $1 \beta$ and IL- 6 were significantly increased in T/HS rats compared with the sham group (Fig. 8). However, PHC treatment prior to or following $\mathrm{T} / \mathrm{HS}$ induction significantly reduced the levels of TNF- $\alpha$, IL-1 $\beta$ and IL-6 compared with the T/HS group.

\section{Discussion}

ALI and ARDS induce a continuum of lung changes arising from a wide variety of lung injuries, causing high morbidity and mortality rates in intensive care units (21). An increasing number of studies have demonstrated the critical role of Fas/FasL activation in pulmonary epithelial cells $(22,23)$. However, little is known about the exact mechanism that underlies this. The present study established a novel double-hit rat model of ALI (blunt trauma followed by hemorrhage shock), induced by T/HS, and determined the effect of PHC on the Fas/FasL signaling pathway by assessing pulmonary apoptosis, inflammation and lung damage. The results indicated that the T/HS-constructed ALI rat model significantly increased arterial hypoxemia, alveolar edema, leucocytosis in the interstitial capillaries and alveolar hemorrhage in histological assessments, which is consistent with a previous study (12). Additionally, it was revealed that $\mathrm{PaO}_{2} / \mathrm{FiO}_{2}$ in $\mathrm{T} / \mathrm{HS}$ rats was $<300$.

It has been demonstrated that a multitude of pathways are involved in the regulation of apoptosis, including Fas/FasL (24). Gil et al (25) indicated that the Fas signaling pathway was associated with ALI severity in mice. In the present study, to determine the involvement of the Fas/FasL signaling pathway in the mediation of lung tissue cell apoptosis, the lungs of rats with ALI induced by T/HS were assessed via TUNEL staining $6 \mathrm{~h}$ following T/HS. The results demonstrated that following T/HS challenge, the expression of Fas, FasL caspase- 3 and caspase- 8 was significantly increased, and the BALF PMN count and protein concentration were markedly increased. The current study therefore hypothesized that Fas/FasL activation resulted in an increase of proinflammatory cytokines (TNF- $\alpha$, IL-6 and IL-1 $\beta$ ) and apoptotic cells in the lungs during T/HS. In this regard, the activation of the Fas/FasL signaling pathway may not only induce apoptosis, but may also lead to the production and secretion of cytokines. Consistent with the findings of Weckbach et al (26), the combination of T/HS induced lung cell apoptosis, lung inflammation, subsequent PMN recruitment and disruption of the alveolocapillary barrier. Additionally, Serrao et al (27) demonstrated that PMNs induce the apoptosis of lung epithelial cells by upregulating FasL. Perl et al (28) also indicated that in the absence of PMNs, blunt chest trauma-induced ALI was mitigated. Therefore, lung cell apoptosis and lung inflammation have been identified as pathophysiologically relevant mechanisms in the development of ALI.

Multiple findings have revealed that $\mathrm{PHC}$ is effective in the treatment of ALI (29,30). Additional studies further indicate that PHC selectively blocks muscarinic acetylcholine (M) receptor M1, M3 and nicotinic acetylcholine receptors, with fewer M2 receptor-associated cardiovascular side effects than hyoscyamine (31). An increasing number of studies have indicated that PHC exhibits anti-apoptotic, anti-inflammatory and anti-oxidative stress effects under organ dysfunction $(16,32,33)$. A previous study demonstrated that PHC exerted anti-inflammatory properties and protective effects during ALI via the inhibition of the toll-like receptor 4 signaling pathway (34). Furthermore, Cao et al (35) confirmed that PHC alleviated cerebral injury by inhibiting the p38 mitogen-activated protein kinase (MAPK) and caspase-3. Wang et al (36) also revealed that the administration of PHC in renal ischemia-reperfusion injured rats decreased the level of malondialdehyde and the expression of p38 MAPK, nuclear factor- $\kappa \mathrm{B}$ and caspase-3 expression, and attenuated the reduction in superoxide dismutase activity. Wu et al (34) clarified that $\mathrm{PHC}$ significantly increased $\mathrm{PaO}_{2}, \mathrm{pH}, \mathrm{PaO}_{2} / \mathrm{FiO}_{2}$ and $\mathrm{PaCO}_{2}$, reduced IL- 6 and IL- $1 \beta$ levels, and reduced pulmonary myeloperoxidase activity in an ALI model induced by lipopolysaccharide.

The results of the current study revealed that PHC treatment prior to or following T/HS induction attenuated lung damage by significantly improving pulmonary oxygenation and by decreasing BALF PMN count and protein concentration. Furthermore, the Fas/FasL signaling pathway, levels of cell apoptosis and the expression of proinflammatory cytokines, including TNF- $\alpha$, IL- 6 and IL-1 $\beta$, were inhibited following PHC treatment. Whether PHC improves the overall survival of rats with ALI was not assessed in the present study and as such, should be a subject of future experiments. Additionally, the exact mechanism of how PHC ameliorates ALI remains poorly understood, which warrants future investigation.

In conclusion, the present study indicates that the Fas/FasL signaling pathway may serve a pivotal role in the pathogenesis of lung injury following T/HS. PHC may also serve a protective 
role in ALI by inhibiting the Fas/FasL signaling pathway. Therefore, PHC may be a potential agent for future treatment of inflammatory diseases, including ALI.

\section{Acknowledgements}

Not applicable.

\section{Funding}

The present study was supported by the National Natural Science Foundation of China (grant no. 81571941) and the Natural Science Foundation of Hubei Province of China (grant no. 2016CFB251).

\section{Availability of data and materials}

The datasets used and/or analyzed during the current study are available from the corresponding author on reasonable request.

\section{Authors' contributions}

XW and XS designed the experiments. XW, QK and WD performed the experiments. WD and LZ analyzed experimental results. XW, QK and XS wrote the manuscript and critically revised it for important intellectual content. All authors have read and approved the final version of the manuscript.

\section{Ethics approval and consent to participate}

Ethics approval was provided by the Medical Ethics Committee of Renmin Hospital of Wuhan University. All surgical procedures were performed in accordance with Wuhan University Animal Care and Use Committee.

\section{Patient consent for publication}

Not applicable.

\section{Competing interests}

The authors declare that they have no competing interests.

\section{References}

1. Liu Y, Du DY, Hu X, Xia DK, Xiang XY, Huang C, Zhou JH and Jiang JX: Prevalence and mortality of severe chest trauma in three gorges area of China. Zhongguo Yi Xue Ke Xue Yuan Xue Bao 34: 567-572, 2012 (In Chinese).

2. Erickson SE, Martin GS, Davis JL, Matthay MA and Eisner MD; NIH NHLBI ARDS Network: Recent trends in acute lung injury mortality: 1996-2005. Crit Care Med 37: 1574-1579, 2009.

3. Gallagher JJ: Management of blunt pulmonary injury. AACN Adv Crit Care 25: 375-386, 2014.

4. Brown LM, Kallet RH, Matthay MA and Dicker RA: The influence of race on the development of acute lung injury in trauma patients. Am J Surg 201: 486-491, 2011.

5. Messer MP, Kellermann P, Weber SJ, Hohmann C, Denk S, Klohs B, Schultze A, Braumüller S, Huber-Lang MS and Perl M: Silencing of fas, fas-associated via death domain, or caspase 3 differentially affects lung inflammation, apoptosis, and development of trauma-induced septic acute lung injury. Shock 39: 19-27, 2013.
6. Thakkar RK, Chung CS, Chen Y, Monaghan SF, Lomas-Neira J, Heffernan DS, Cioffi WG and Ayala A: Local tissue expression of the cell death ligand, fas ligand, plays a central role in the development of extrapulmonary acute lung injury. Shock 36: 138-143, 2011.

7. Glavan BJ,Holden TD, Goss CH, Black RA, Neff MJ,Nathens AB, Martin TR and Wurfel MM; ARDSnet Investigators: Genetic variation in the FAS gene and associations with acute lung injury. Am J Respir Crit Care Med 183: 356-363, 2011.

8. Herrero R, Tanino M, Smith LS, Kajikawa O, Wong VA, Mongovin S, Matute-Bello G and Martin TR: The Fas/FasL pathway impairs the alveolar fluid clearance in mouse lungs. Am J Physiol Lung Cell Mol Physiol 305: L377-L388, 2013.

9. Wang Z, Lin D, Zhang L, Liu W, Tan H and Ma J: Penehyclidine hydrochloride prevents anoxia/reoxygenation injury and induces H9c2 cardiomyocyte apoptosis via a mitochondrial pathway. Eur J Pharmacol 797: 115-123, 2017.

10. Yang Y, Zhao L and Ma J: Penehyclidine hydrochloride preconditioning provides cardiac protection in a rat model of myocardial ischemia/reperfusion injury via the mechanism of mitochondrial dynamics mechanism. Eur J Pharmacol 813: 130-139, 2017.

11. Zhu R, Zhao Y, Li X, Bai T, Wang S, Wang W and Sun Y: Effects of penehyclidine hydrochloride on severe acute pancreatitis-associated acute lung injury in rats. Biomed Pharmacother 97: 1689-1693, 2018.

12. Wu XJ, Liu HM, Song XM, Zhao B, Leng Y, Wang EY, Zhan LY, Meng QT and Xia ZY: Penehyclidine hydrochloride inhibits TLR4 signaling and inflammation, and attenuates blunt chest trauma and hemorrhagic shock-induced acute lung injury in rats. Mol Med Rep 17: 6327-6336, 2018.

13. Zheng F, Xiao F, Yuan QH, Liu QS, Zhang ZZ, Wang YL and Zhan J: Penehyclidine hydrochloride decreases pulmonary microvascular endothelial inflammatory injury through a beta-arrestin-1-dependent mechanism. Inflammation 41: 1610-1620, 2018.

14. Wang LL, Zhan LY, Wu XJ and Xia ZY: Effects of penehyclidine hydrochloride on apoptosis of lung tissues in rats with traumatic acute lung injury. Chin J Traumatol 13: 15-19, 2010.

15. Cui J, Li CS, He XH and Song YG: Protective effects of penehyclidine hydrochloride on acute lung injury caused by severe dichlorvos poisoning in swine. Chin Med J (Engl) 126: 4764-4770, 2013

16. Wu XJ, Xia ZY, Wang LL, Luo T, Zhan LY, Meng QT and Song XM: Effects of penehyclidine hydrochloride on pulmonary contusion from blunt chest trauma in rats. Injury 43: 232-236, 2012.

17. Raghavendran K, Davidson BA, Helinski JD, Marschke CJ, Manderscheid P, Woytash JA, Notter RH and Knight PR: A rat model for isolated bilateral lung contusion from blunt chest trauma. Anesth Analg 101: 1482-1489, 2005.

18. Wu X, Song X, Li N, Zhan L, Meng Q and Xia Z: Protective effects of dexmedetomidine on blunt chest trauma-induced pulmonary contusion in rats. J Trauma Acute Care Surg 74: 524-530, 2013.

19. Kellner M, Noonepalle S, Lu Q, Srivastava A, Zemskov E and Black SM: ROS signaling in the pathogenesis of Acute Lung Injury (ALI) and Acute Respiratory Distress Syndrome (ARDS). Adv Exp Med Biol 967: 105-137, 2017.

20. Wu DQ, Wu HB, Zhang M and Wang JA: Effects of zinc finger protein A20 on Lipopolysaccharide (LPS)-induced pulmonary inflammation/anti-inflammatory mediators in an acute lung injury/acute respiratory distress syndrome rat model. Med Sci Monit 23: 3536-3545, 2017.

21. Agarwal R, Handa A, Aggarwal AN, Gupta D and Behera D: Outcomes of noninvasive ventilation in acute hypoxemic respiratory failure in a respiratory intensive care unit in north India. Respir Care 54: 1679-1887, 2009.

22. Han F, Luo Y, Li Y, Liu Z, Xu D, Jin F and Li Z: Seawater induces apoptosis in alveolar epithelial cells via the Fas/FasL-mediated pathway. Respir Physiol Neurobiol 182: 71-80, 2012.

23. Seitz DH, Palmer A, Niesler U, Braumüller ST, Bauknecht S, Gebhard F and Knöferl MW: Altered expression of Fas receptor on alveolar macrophages and inflammatory effects of soluble Fas ligand following blunt chest trauma. Shock 35: 610-617, 2011.

24. Kobata T, Takasaki K, Asahara H, Hong NM, Masuko-Hongo K, Kato T, Hirose S, Shirai T, Kayagaki N, Yagita H, et al: Apoptosis with FasL+ cell infiltration in the periphery and thymus of corrected autoimmune mice. Immunology 92: 206-213, 1997. 
25. Gil S, Farnand AW, Altemeier WA, Gill SE, Kurdowska A, Krupa A, Florence JM and Matute-Bello G: Fas-deficient mice have impaired alveolar neutrophil recruitment and decreased expression of anti-KC autoantibody: KC complexes in a model of acute lung injury. Respir Res 13: 91, 2012.

26. Weckbach S, Hohmann C, Braumueller S, Denk S, Klohs B, Stahel PF, Gebhard F, Huber-Lang MS and Perl M: Inflammatory and apoptotic alterations in serum and injured tissue after experimental polytrauma in mice: Distinct early response compared with single trauma or 'double-hit' injury. J Trauma Acute Care Surg 74: 489-498, 2013.

27. Serrao KL, Fortenberry JD, Owens ML, Harris FL and Brown LA: Neutrophils induce apoptosis of lung epithelial cells via release of soluble Fas ligand. Am J Physiol Lung Cell Mo Physiol 280: L298-L305, 2001.

28. Perl M, Hohmann C, Denk S, Kellermann P, Lu D, Braumüller S, Bachem MG, Thomas J, Knöferl MW, Ayala A, et al: Role of activated neutrophils in chest trauma-induced septic acute lung injury. Shock 38: 98-106, 2012.

29. Li H, Qian Z, Li J, Han X and Liu M: Effects of early administration of a novel anticholinergic drug on acute respiratory distress syndrome induced by sepsis. Med Sci Monit 11: BR319-BR325, 2011.

30. Shen W, Gan J, Xu S, Jiang G and Wu H: Penehyclidine hydrochloride attenuates LPS-induced acute lung injury involvement of NF-kappaB pathway. Pharmacol Res 60: 296-302, 2009.

31. Han XY, Liu H, Liu CH, Wu B, Chen LF, Zhong BH and Liu KL: Synthesis of the optical isomers of a new anticholinergic drug, penehyclidine hydrochloride (8018). Bioorg Med Chem Lett 15: 1979-1982, 2005.
32. Tan H, Chen L and Ma J: Penehyclidine hydrochloride post-conditioning reduces ischemia/reperfusion-induced cardiomyocyte apoptosis in rats. Exp Ther Med 14: 4272-4278, 2017.

33. Wang D, Jiang Q and Du X: Protective effects of scopolamine and penehyclidine hydrochloride on acute cerebral ischemia-reperfusion injury after cardiopulmonary resuscitation and effects on cytokines. Exp Ther Med 15: 2027-2031, 2018.

34. Wu GM, Mou M, Mo LQ, Liu L, Ren CH, Chen Y and Zhou J: Penehyclidine hydrochloride postconditioning on lipopolysaccharide-induced acute lung injury by inhibition of inflammatory factors in a rodent model. J Surg Res 195: 219-227, 2015.

35. Cao HJ, Sun YJ, Zhang TZ, Zhou J and Diao YG: Penehyclidine hydrochloride attenuates the cerebral injury in a rat model of cardiopulmonary bypass. Can J Physiol Pharmacol 91: 521-527, 2013.

36. Wang YP, Li G, Ma LL, Zheng Y, Zhang SD, Zhang HX, Qiu M and Ma X: Penehyclidine hydrochloride ameliorates renal ischemia-reperfusion injury in rats. J Surg Res 186: 390-397, 2014.

This work is licensed under a Creative Commons Attribution-NonCommercial-NoDerivatives 4.0 International (CC BY-NC-ND 4.0) License. 